\title{
Prenatal solvent exposure association with motor inhibition functional MRI at the age of 10-12 in the PELAGIE mother-child cohort
}

\author{
Anne-Claire Binter*, Inserm U1085 IRSET, Rennes, France \\ Elise Bannier, Inserm U1228 Inria Visages, University Hospital of Rennes, University of Rennes 1, Rennes, France \\ Dave Saint-Amour, Department of Psychology, Université du Québec à Montréal, Montréal, Canada \\ Gregory Simon, CNRS U3521, University of Caen Basse-Normandie, Caen, France \\ Jean-Christophe Ferré, Inserm U1228 Inria Visages, Department of Neuroradiology, University Hospital of Rennes, Rennes, \\ France \\ Christian Barillot, Inserm U1228 Inria Visages, CNRS U6074 IRISA, University of Rennes 1, Rennes, France \\ Jean-François Viel, Inserm U1085 IRSET, University of Rennes 1, Rennes, France \\ Sylvaine Cordier, Inserm U1085 IRSET, Rennes, France \\ Cécile Chevrier, Inserm U1085 IRSET, Rennes, France \\ Fabienne Pelé, Inserm U1085 IRSET, University of Rennes 1, Rennes, France
}

\section{Background/Aim}

Epidemiological studies reported that prenatal solvent exposure was associated with adverse neurobehavioral outcomes that suggest impaired motor inhibition function. Using functional Magnetic Resonance Imaging (fMRI) among children aged 10-12 years old, we aimed at investigating the effect of prenatal exposure to solvents on the activation of cerebral regions involved in the motor inhibition function.

\section{Methods}

Fifty-three children from the PELAGIE mother-child cohort (France, from 2002) underwent a 10-minutes fMRI go/nogo task using an event-related design. : Children were asked to press a button as quickly as possible in response to a green smiley ('go') but not press the button when seeing a red smiley ('no-go': inhibition event). Solvent exposure was assessed at the beginning of pregnancy ( $<19 \mathrm{WG}$ ) by measuring three glycol ether metabolites in maternal urine and defined as 'unexposed' ( $1^{\text {st }}$ tercile) or 'exposed' ( $2^{\text {nd }}$ and $3^{\text {rd }}$ tercile) for each solvent. Go and nogo cerebral activations at individual and group levels were assessed using SPM. T-test with p-value corrected for multiple testing was used to estimate difference of brain activations between differentially exposed children. Confounders as tobacco smoke and alcohol consumption at the beginning of pregnancy were taken into account by restriction.

Results

'No-go' cerebral activations were larger in middle and inferior frontal regions of the right hemisphere among children unexposed to methoxyacetic acid as compared to exposed ones. No differential activations were found with ethoxyacetic acid and phenoxyacetic acid.

\section{Conclusions}

The present study suggests association between prenatal solvent exposure and larger activations in cerebral regions involved in motor inhibition function at the age of 10-12 years old. Analysis will be extended to a bigger sample (on-going urinary measurements) to confirm and refine these results. 